Laboratory, L.N. Pfeiffer of Bell Laboratories/Lucent Technologies, and their colleagues have created a device that effectively splits a stream of quantum objects such as electrons into two streams according to the spin of each, herding those with "up" spin in one direction and corralling those that spin "down" in another (see Figure 1). By producing such spin-polarized streams, the device could become a key component in quantum computers, which have not yet left the drawing boards of the computer industry but are highly sought after for their purported facility at cracking codes and searching large databases.

"We have achieved spatial spin separation of the holes in gallium arsenide, the spaces that electrons leave behind as they travel through this semiconductor," said Rokhinson, who is an assistant professor of physics in the School of Science. "These holes also have spin characteristics, just as particles do, and separating them according to their spin has been a great challenge. Producing this effect will be critical for the success of any spin-based electronic device, and this separation method could be one of the missing links necessary for the development of quantum computers and nonvolatile memory devices."

Two particles' respective spins, which are opposite but inextricably linked, allow them to form a quantum bit, or qubit, that can actually be in a superposition state that simultaneously represents the possibility of "on" and "off" states, or function as both a one and a zero during digital calculations. This ability to represent two conditions at once, multiplied many times over within a computer chip

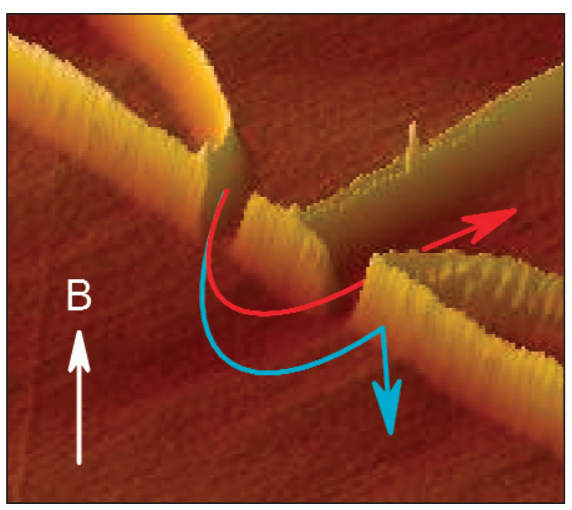

Figure 1. Atomic force micrograph of a device used to separate quantum objects according to their spin. The light-colored lines are oxide separating different regions of two-dimensional "hole gas" beneath the surface. (Graphic courtesy of Purdue University.) that uses a large number of qubits, could be a powerful tool for sifting through information.

"The trouble is, you have to find a way to measure the final quantum state of the qubit after the calculations have been made to extract useful information from them," Rokhinson said. "Only once you have separated them can you obtain the answer to your calculations. This measurement issue has been one of the big challenges of the field."

Part of the reason behind this difficulty lies in the very weak coupling of spin with the environment. In semiconductor materials, Rokhinson said, spin is coupled many trillions of times less than charge is, and spin experiences comparatively little influence from nearby matter.

"In practical terms, this means you can try to make a particle flip its spin from 'up' to 'down,' but it won't feel you pushing," he said. "Researchers have tried to polarize the particles using everything from light waves to strong magnetic fields, but nothing was working well enough to separate them."

However, Rokhinson's team discovered that semiconductors made of highly purified GaAs sandwiched between layers of $\mathrm{AlGaAs}$ possessed a natural property that when harnessed could push the holes into two different directions according to their spin state, as the researchers reported in the October 1 issue of Physical Review Letters (146601).

Using a so-called magnetic focusing technique, a small perpendicular magnetic field bends a beam of holes in a GaAs semiconductor along two different cyclotron trajectories, with the radius depending on the spin of the particles. Those holes with "up" spin curve in one direction, those with "down" spin in the other.

Rokhinson said, "The only reason I have used holes rather than electrons is a pure convenience: GaAs/AlGaAs heterostructures are the cleanest systems we can grow, and two-dimensional gases of this material have the highest mobility (the catch is that our particles should not experience any scattering while completing the half-a-circle, which is a few microns)." Spin-orbit interaction for electrons in GaAs is an order of magnitude weaker than for holes. "But the physics is the same," said Rokhinson. "As long as we deal with charged particle with spinorbit coupling we can construct a 'spin spectrometer' of a 'spin filter.'"

\section{Nanoporous Structures Formed by Electrodeposition Process}

Existing techniques for producing functionally graded electrodes require the deposition of multiple layers of material, each with a different pore structure. Each layer must be thermally and chemically compatible and able to conduct electricity. M. Liu of the Georgia Institute of Technology and collaborators H.-C. Shin and J. Dong have found a simpler means of producing three-dimensional nanoporous electrodes that facilitate the movement of liquids and gases.

By generating hydrogen bubbles during the deposition of copper, tin, or a coppertin alloy onto a copper substrate, the researchers create self-supported metallic foam electrodes that contain a complex network of interconnected pores. Because the bubbles expand as they move away from the substrate, they create passageways through the deposited metal that become wider the closer they get to the outside of the electrode.

The tapered passageways should allow gases and fluids to move more easily through these functionally graded electrodes, enhancing the performance of solid-oxide fuel cells, lithium batteries, and chemical sensors, for example. The nanoporous nature of the structures provides a large surface area on which electrochemical reactions can take place.

"By adjusting the properties of the electrolyte- the viscosity and chemical composition-we can change the size of the gas bubbles we generate," said Liu, a professor in the School of Materials Science and Engineering. "Getting the bubbles small enough allows us to produce threedimensional nanostructures in which the pores are small on the inside but taper to larger pores on the outside."

Lui, who is also the co-director of Georgia Tech's Center for Innovative Fuel Cell and Battery Technologies, said, "In our electrode, the gradient is created naturally and is ideal for our needs.... You can avoid the complexity of creating multiple layers."

The researchers said that the production of hydrogen bubbles serves as the basic sculpting tool for creating the pore structure. The gas acts as a dynamic template for the formation of the structure and serves as a barrier for the diffusion of reactive ions from the electrolyte to regions around the branches that are depleted of ions, preventing overgrowth of passageways.

The new electrodes vary in thickness from a few microns up to $15 \mu \mathrm{m}$, depending on the materials used and the processing time. Liu expects that copper-based electrodes will be useful in solid-oxide fuel cells, while tin-based electrodes will be useful in lithium batteries.

Microscope study reveals subtle differ- 
ences between electrodes made from copper and those made from tin. The branches of the tin deposits are longer and straighter than those of copper. The copper foams also contain nanometer- sized grains and pores not seen in the tin structures. Liu said the differences may be related to the fact that during deposition of the copper electrodes, gas bubbles are produced from both the deposited copper and the substrate. In the tin electrodes, only the substrate produces bubbles. The researchers will publish their work in an upcoming issue of Advanced Functional Materials.

\section{News of MRS Members/Materials Researchers}

S. Banerjee has been appointed director of the Bhabha Atomic Research Centre (BARC), Mumbai, India, beginning his tenure in April.

Chad Mirkin, the George B. Rathmann Professor of Chemistry and Director of the Institute for Nanotechnology at Northwestern University, has been selected to receive the National Institutes of Health (NIH) Director's Pioneer Award in recognition of his efforts in pioneering the development of nanoscale chemical and biological sensors. Mirkin is also the founder of Nanosphere, Inc. in Northbrook, Ill. and NanoInk, Inc. in Chicago, Ill.

Baldev Raj has been appointed direc- tor of the Indira Gandhi Centre for Atomic Research (IGCAR), Kalpakkam, India, beginning his tenure in May.

Matthew Tirrell, Dean of the College of Engineering at the University of California, Santa Barbara, has been appointed as a Venture Partner at NGEN Partners LLC (Santa Barbara, Calif.), a venture capital fund with a focus on the materials field.

Chris G. Van de Walle has joined the Materials Department at the University of California, Santa Barbara as professor of computational materials. He will also be associated with the Materials Research Laboratory and the California NanoSystems Institute.
Jennifer West, the Isabel C. Cameron Professor of Bioengineering and professor of chemical engineering at Rice University, has been selected to receive the 2004 Annunzio Award from the Christopher Columbus Fellowship Foundation in recognition of her work in biomaterials and tissue engineering.

Wolfgang Windl of The Ohio State University and Gerd Duscher of North Carolina State University have been awarded the 2004 Nano Technology Industrial Impact Award from the Nano Science and Technology Institute for their discovery of atomically sharp "perfect" interfaces in $\mathrm{Si}: \mathrm{Ge} / \mathrm{SiO}_{2}$ devices.

\section{News of MRS Corporate Affiliates/Materials Institutions}

The Aachen University of Technology, AIXTRON AG (Aachen, Germany), and Philips Lighting (AachenRothe Erde, Germany) have signed a collaboration agreement aimed at the joint development of novel large-area white organic light-emitting diodes (OLED) (www.rwth-aachen.de; www.aixtron.com; www.lighting.philips. com).

The Ceramics and Composites Laboratory has been launched within the Department of Engineering Materials at Sheffield University, United Kingdom, to develop new materials. The laboratory has been guaranteed funding of $£ 6$ million over the next five years from the Engineering and Physical Research Council (EPSRC) (www.shef.ac.uk/materials/ research/centres/ccl.html).

The Institute for Lasers, Photonics and Biophotonics at the State University of New York at Buffalo has received a $\$ 925,000$ grant from the John R. Oishei Foundation for research and development in nanomedicine and nanobiotechnology (wwww.photonics.buffalo.edu).

Matsushita Electric Industrial Co. Ltd. (Osaka, Japan) has joined the International SEMATECH Manufacturing Initiative (ISMI), becoming the newest member of the global consortium of leading semiconductor manufacturers engaged in cooperative precompetitive work to improve semi- conductor manufacturing productivity in the current and future manufacturing facilities of its member companies. ISMI is a wholly-owned subsidiary of SEMATECH, Inc. (Austin, Texas). Matsushita joins AMD, Hewlett-Packard, IBM, Infineon, Intel, Freescale, Philips, Texas Instruments, and TSMC (www.panasonic.co.jp/global; www.ismi.sematech.org).

NanoDynamics (Buffalo, N.Y.) has signed a joint venture agreement with the New Zealand-based technology company, Nano Cluster Devices Ltd. (NCD) to commercialize NCD's technology process for self-assembly of nanowires in production of semiconductors and electronic components (www.nanodynamics.com; www. nanoclusterdevices.com).

Nanoscale Science and Engineering Centers have been announced by the National Science Foundation for the following universities: University of California-Berkeley, Center of Integrated Nanomechanical Systems (\$11.9 million), director: Alex Zettl; Northeastern University, Center for High Rate Nanomanufacturing (\$12.4 million), director: Ahmed Busnaina; Ohio State University, Center for Affordable Nanoengineering of Polymer Biomedical Devices (\$12.9 million), director: L. James Lee; University of Pennsylvania, Center on Molecular Function at the Nano/Bio Interface (\$11.4 million), director: Dawn Bonnell; Stanford University, Center for Probing the Nanoscale (\$7.5 million), director: Kathryn Moler; University of Wisconsin-Madison, Center for Templated Synthesis and Assembly at the Nanoscale (\$13.4 million), director: Paul Nealey (wwww.nsf.gov).

The Northwestern Institute on Complex Systems (NICO) was established at Northwestern University (Evanston, Ill.) to stimulate path-breaking research of complex systems across traditional boundaries. NICO comprises a distinguished and diverse group of faculty from all areas of the university, including engineering, business, natural sciences, education, medicine, law, and the social sciences. Mark A. Ratner, Morrison Professor of Chemistry at Northwestern, is the group leader of the NICO-affiliated center on materials selfassembly processes (www.northwestern. edu/research/nico).

\footnotetext{
Correction

In the article, "Novel Materials and Applications of Electronic Noses and Tongues," published in the October 2004 issue of MRS Bulletin, the publication listed as Reference 26 was incorrect. The correct reference is: N.A. Rakow and K.S. Suslick, "A Colorimetric Sensor Array for Odour Visualization," Nature 406 (2000) p. 710.
} 